\title{
Functional analysis of the surface of implants in the interaction with cells: a direct viability and cell compatibility analysis
}

Matos $\mathrm{PC}^{*}$, Gehrke $\mathrm{SA}^{* \star}$, Cavalcanti Lima $\mathrm{JH}^{*}$, Tanaka $\mathrm{M}^{*}$, Maia MC $\mathrm{C}^{*}$, Pérez-Díaz $\mathrm{L}^{\star * *}$.

*Universidade de Guarulhos, Guarulhos, Brazil;; **Biotecnos - Technology and Science, Montevideo, Uruguay; Universidad de la Republica, Montevideo, Uruguay.

\section{Abstract}

Objectives: To evaluate the effect of topography in nanoscale, titanium surfaces were bombarded by argon ions (a chemically inert gas), in an atmosphere of plasma. The effects of surface parameters on morphology, adhesion, proliferation, and MC3T3-E1 preosteoblasts differentiation were analyzed. Nontreated (smooth) surfaces were used as a control.

Materials and Methods: Cells were cultivated over the implants surface, MC3T3-E1 cells were grown on the different substrates (PT and PTCA) for $24 \mathrm{~h}$ at the density of $2 \times 103$ cells/disk: uncoated (PT) and nano-coated (PTCA) machined discs. They were then fixed in karnovsky, dehydrated and dried with hexamethyl (HMDS), processed to MEV and analyzed under a Labnano JEOL microscope. This assay was analyzed by fluorescence microscopy from two cells that simultaneously determine living and dead cells of two probes, based on the measurement of intracellular parameters of cell viability through esterase activity and integrity analysis of the plasma membrane. The probes used are: green cells to live cells and the ethidium that marks red for cells dead marking with ethidium is excluded by the presence of membrane plasma levels of viable cells. In this way, the determination of the cell viability in this assay depends on the physical and biochemical properties of the cell.

Results: Observe the negative control (C-), which represents viable cells adhered on glass substrate, biocompatible under normal culture conditions. Both the negative control and the PT and PTCA samples present an obvious labeling with green calcein, confirming the viability of the adhered cells on the surface of the respective substrates. In contrast to the positive control $\left(\mathrm{C}_{+}\right)$, represented by cells challenged with dimethyl sulfoxide (DMSO) at 10\% concentration for 2 hours before the assay, which presented cellular viability impairment evidenced by the red tagged ethidium tag. The samples PT 6002 (machined) exhibit morphology with the presence of structures of recognition and adhesion to the filopodia substrate. Cell spreading is also observed in cells adhered to the PTCA 6002 (nano) surface, however, note the difference in the level of spreading

Conclusions: Within the limitations of this study, the results indicate that the study samples showed no evidence of cellular toxicity. The results show cells adhered in substrate with and without nano treatment. In cells that received nano coating, surface area is higher, as is the level of interaction with the substrate, extremely adjacent to the PTCA surface. The absence of filipodia in the PTCA samples is not relevant, due to the stage of interaction in which the cells are adhered to this type of substrate.

\section{Background and Aim}

In the human environment, the surface of the implant material makes the first contact with cells, which normally adhere to the material through adhesive cell proteins such as integrin, fibronectin, vitronectin, and collagen. Reactions between the metallic implant surface and the cells are important because the biomolecules that migrate to the implant possibly form a protective layer and in turn influence the cell's capacity to proliferate and differentiate. On the other hand, body fluid can dissolve metal ions from the implant resulting in corrosion products which may show toxicity when they interact with biomolecules and cells.

\section{Methods and Materials}

Cells were cultivated over the implants surface, MC3T3-E1 cells were grown on the different substrates (PT and PTCA) for 24h at the density of 2x103 cells/disk: uncoated (PT) and nano-coated (PTCA) machined discs. They were then fixed in karnovsky, dehydrated and dried with hexamethyl (HMDS), processed to MEV and analyzed under a Labnano JEOL microscope.

PT surface

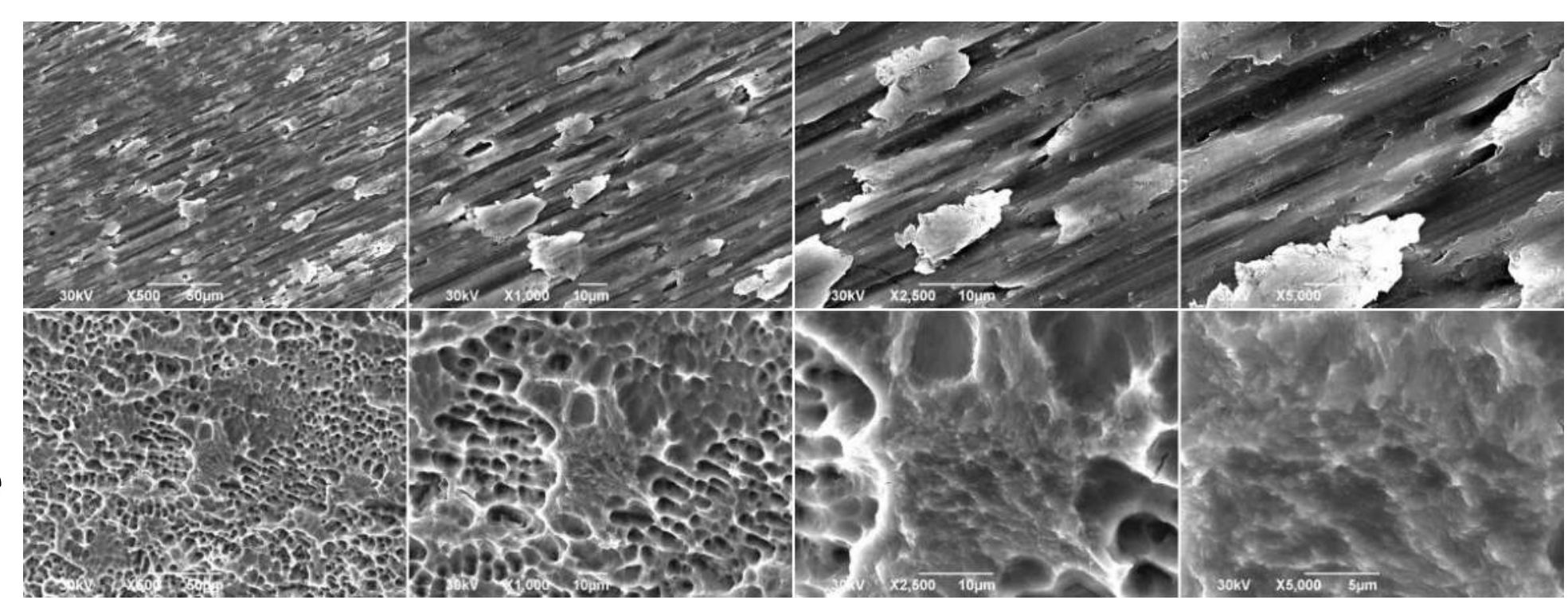

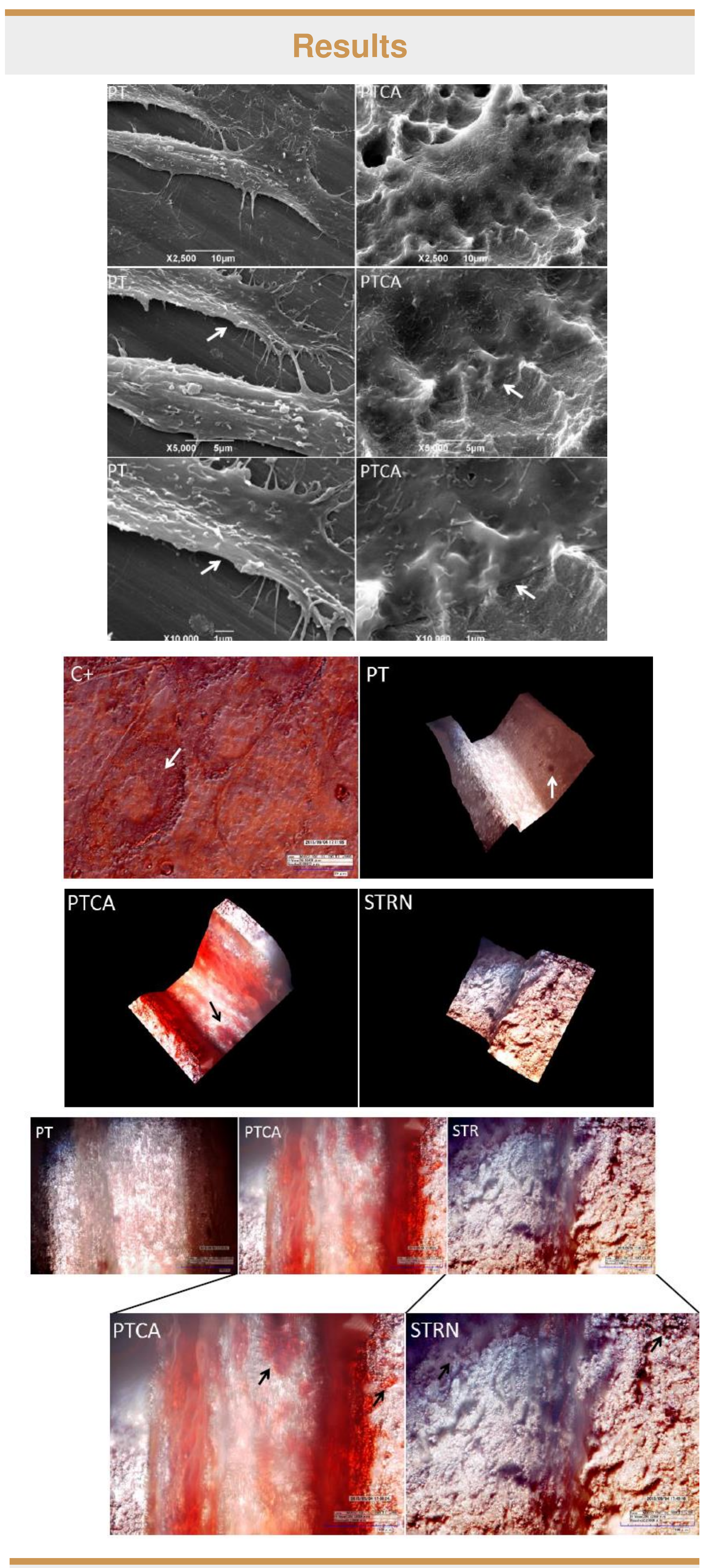

Conclusions

Within the limitations of this study, the results indicate that the study samples showed no evidence of cellular toxicity. The results show cells adhered in substrate with and without nano treatment. In cells that received nano coating, surface area is higher, as is the level of interaction with the substrate, extremely adjacent to the PTCA surface.

\section{References}

1. D. M. Dohan Ehrenfest, P.G. Coelho, B.-S. Kang, Y.-T. Sul, and T. Albrektsson, "Classification of osseointegrated implant surfaces: materials, chemistry and topography," Trends in Biotechnology, 2010.

2. G. Mendonc sa, D. B. S. Mendonc sa, F. J. L. Arag̃ao, and L. F. Cooper, "Advancing dental implant surface technology-from micron- to nanotopography," Biomaterials, 2008.

3. L. Guida, A. Oliva, M. A. Basile, M. Giordano, L. Nastri, and M. Annunziata, "Human gingival fibroblast functions are stimulated by oxidized nano-structured titanium surfaces," Journal of Dentistry,2013. 